AperTO - Archivio Istituzionale Open Access dell'Università di Torino

\title{
Body Image and Binge Eating Disorder
}

\section{This is a pre print version of the following article:}

Original Citation:

\section{Availability:}

This version is available http://hdl.handle.net/2318/1758287

since 2020-10-13T17:22:01Z

Publisher:

Springer

Published version:

DOI:10.1007/978-3-319-90817-5_10

Terms of use:

Open Access

Anyone can freely access the full text of works made available as "Open Access". Works made available under a Creative Commons license can be used according to the terms and conditions of said license. Use of all other works requires consent of the right holder (author or publisher) if not exempted from copyright protection by the applicable law. 


\title{
Body image and binge eating disorder (chapter 17)
}

\author{
Federico Amianto, Secondo Fassino
}

\subsection{Introduction}

\subsubsection{Diagnostic criteria of Binge Eating Disorder}

The recently published 5th revision of the Diagnostic and Statistical Manual of Mental Disorders (DSM-5) includes binge eating disorder (BED) for the first time as a diagnostic category in the Eating Disorders section, while before it was listed as an Eating Disorder not Otherwise Specified (EDNOS) needing future definition. According to the criteria, which are basically in line with the former research criteria of the DSM-IV-TR, BED is characterized by recurring episodes of binge eating over the course of at least 3 months with one binge episode on average per week (DSM-IV-TR: duration of 6 months with two binge episodes per week), in which a large amount of food is consumed in a distinct amount of time, often accompanied by a feeling of loss of control. To measure severity of the disorder, the DSM-5 proposes binge eating frequency as a specifying criterion. In contrast to other prominent eating disorders, such as Anorexia nervosa ( $\mathrm{AN}$ ) or Bulimia nervosa $(\mathrm{BN})$, no regular inappropriate compensatory behaviors are undertaken to avoid weight gain, e.g. self-induced vomiting, abuse of laxatives or excessive sport or exercise. Binge eating is often associated with eating quickly and to the point of feeling uncomfortably full, along with feelings of disgust and shame, leading to the individual mostly eating alone. Whereas in the diagnostic criteria for $\mathrm{AN}$ and $\mathrm{BN}$, body image disturbance is a required criterion for diagnosis, it is not included in the criteria for BED. However, there is growing evidence that body image disturbance might be characteristic for BED as well even though only a limited number of studies have addressed body image disturbance in BED (1).

\subsubsection{Epidemiology and comorbidity of BED}

BED is a relatively common disorder, with an estimated lifetime prevalence in general population around $1,4 \%$. This disease is of particular interest for its frequency in primary care, its link with obesity and its medical and psychiatric comorbidities, leading to high socio-economic impact due to reduced quality of life and increased health service utilization (2,3). As a consequence of the excessive food intake, the most frequent comorbidity in BED is obesity, with about $40-70 \%$ of people with BED also suffering from severe overweight. Prevalence is likely to increase, especially because of the rising incidence that both obesity and EDs show worldwide rather than for the widening of diagnostic criteria introduced in DSM-5 (1,4,5).

Furthermore, BED is associated with elevated eating disorder psychopathology, increased body dissatisfaction and decreased self-esteem. Clinical studies indicate that among obese individuals, those with BED experience greater eating disorder psychopathology, psychiatric and medical comorbidity, impairment in social and occupational functioning, and reduced quality of life than those without BED $(6,7,8)$.

Despite the fact that obese subjects with and without BED do not differ in degree of overweight, binge eaters show considerable more eating, weight, and shape concerns, and also lower levels of self-reported physical appearance and global self-worth. Dietary restraint is generally assumed to be a core characteristic of binge eating among individuals with eating disorders and a pathogenic element of the binge behavior itself (9). It remains unclear to what extent dietary restraint has a function in the binge eating problems among obese subjects (10).

\subsubsection{Assessment of BED}


The diagnosis of BED is eminently clinical and should be performed by an expert psychiatrist, possibly using a semi-structured interview such as the SCID (Structured Clinical Interview) for the DSM 5 criteria. Nevertheless in clinical studies the BED is quantified through various tools:

- Eating Disorder Examination-Questionnaire (EDE-Q) (11), a self-report questionnaire that assesses the frequency of key eating disorder behaviors, differentiating between the various forms of overeating and providing the determination of binge eating as defined in the $4^{\text {th }}$ edition of the Diagnostic and Statistical Manual of Mental Disorders (DSM-IV) (10,12);

- Binge Eating subscale of the Minnesota Eating Behavior survey (13), which assesses the presence or absence of binge eating symptoms, such as feeling out of control while eating and eating when upset, not captured by the EDE-Q binge eating frequency item (6);

- Dutch Eating Behavior Questionnaire (DEBQ) (14), auto administered, designed to investigate disordered eating behaviours in three different sub-domains: restrained eating, emotional eating, external eating.

\subsection{Body image and the concept of Self}

The self, according to the model proposed by Amianto and coworkers (15), may be understood as an integrative structure of the mind that organizes and coordinates different functions (affective, cognitive, social, sensorimotor, and vegetative) with regard to interoceptive and exteroceptive stimuli from one's own body and the environment. In this model, the self is the cognitive, conative, and affective representation of one's subjective experience and one's identity. Thus, the self plays an integral part in human motivation, cognition, affect, and social identity. The self also implies a temporal concept. It extends across time from the past over the present and to the future. In contrast to the concept of identity, which describes the diachronic sameness of a person over time, the concept of self is synchronic, that is, it concerns the experience of an individual as him or herself, and allows him or her to attribute specific experiences, persons, or objects at a particular point in time. This entails a self-specific organization of both neural and psychological activity (16). According to Higgins (17), our behavior is guided by three different domains of the self: the "actual" self (what we think we are), the "ideal" self (what we want to be) and the "ought" self (what we think we ought to be with regards to social norms). The actual self represents our core self-concept. It describes the person we think we are, with all its attributes and traits. The ideal and ought selves are self-guides, in that they drive us to seek new self-states. Whereas the ideal self represents what we would ideally like to be and contains the traits and characteristics of that ideal person, the ought self represents the kind of person we think we need to become if we are to comply with social norms. The ought self therefore contains traits and characteristics that are highly normative and socially constrained. Higgins' theory suggests that people are motivated to achieve a certain match between their self-concept and their self-guides. Any perceived discrepancy between the self-concept and a self-guide leads to different negative emotional and motivational states, depending on the type of discrepancy. Actual/ideal self-discrepancies result in disappointment or dissatisfaction, while actual/ought self-discrepancies lead more to feelings of fear and threat. Selfdiscrepancies, assessed with Higgins' method are related to body dissatisfaction and disordered eating, with the actual/ideal discrepancy being related to bulimia and the actual/ought discrepancy to anorexia. High self-discrepancies are also associated with low self-esteem and emotional vulnerability (18).

Often in the BED subjects it emerges another problem relating the self and body image: the body image is not completely integrated in the Self. BED and in general obese subject do not completely or stably diachronically perceive their body image. It may happen that even openly recognizing of being overweight they intimately deny their body shape and pretend to be thinner than they really are (19). Thus they may also engage in activities that are not adequate for their body shape or weight (such as dressing inappropriately, trying to pass through small spaces or attempting risky sports). In particular when they are overwhelmed by emotional eating they completely loose the 
contact with their sense of being fat and the need to contain their impulses and this reduces the ability to contain their behaviors. Also after their bingeing they do not perceive the need in engaging in compensatory behaviors as BN subjects. They protect themselves for the painful feeling of being overweight removing the perception of the body self, sometimes refusing to face with the mirrors, to speak about the fat as a serious problem and in general escaping from the awareness of their condition.

At a deeper layer the BED body image perception may be significantly hampered by the difficulty of these subjects of setting, perceiving and maintaining the boundaries of their Self. They in general express poor skills in setting and respecting the limits in general, also with respect to emotions, relationships, social cues and requests. BED individuals often shift towards an attitude of requesting unlimited emotional expression, relational gratification, love and social recognition. This is expressed by their unlimited attitudes towards food and visualized by their limitless growth in body shape (20-23).

\subsubsection{Overvaluation of weight and shape}

Overvaluation of body shape/weight refers to one's preoccupation with shape and weight and using one's shape and weight as a major source of self-esteem (24). In the transdiagnostic cognitive behavioral model of eating disorders (25), overvaluation is the core psychopathology that maintains eating disorder symptoms and behaviors. According to Fairburn, shape/weight concerns, such as feelings of fatness (feeling fat or that a specific body part is fat) and fat phobia (fear of becoming fat), are conceptualized as distinct constructs that emanate from overvaluation of shape/weight (26).

It has been shown that overvaluation of weight and shape seems to occur to a comparable degree in BED as in $\mathrm{AN}$ and $\mathrm{BN}$. When comparing obese persons with BED to individuals with obesity without an eating disorder a number of studies reported significantly higher weight and shape concerns in the first (it is present in $60 \%$ of BED patients, a level which is significantly higher than in non-BED obese patients, even though it is lower than in $\mathrm{AN}$ and $\mathrm{BN}$, where it is nearly universal). Even if this feature is present only in a subgroup of BED patients, and so it is not included among the official diagnostic criteria, it is related to higher psychosocial impairment and worse quality of life (27).

A stronger overvaluation of shape and weight also seems to be associated with a more pronounced eating disorder pathology in general as well as with higher levels of depression in BED and is shown to act as a mediator between weight bias internalization and self-esteem. Overvaluation of shape and weight can be thus considered either a diagnostic specifier or a dimensional severity rate, with a primary relevance for diagnosis, treatment choice, global impairment and outcome, and it can also be considered as a specific target for psychological therapies (1,27-29). Furthermore, it has been shown that overvaluation of body shape and weight predicts lower rates of remission from binge eating and higher frequency of binge eating at 12-month follow-ups after completing treatments (28).

\subsubsection{How overvaluation of weight and shape may lead to binge-eating}

The manner by which overvaluation of body shape and weight maintains eating disorders is complex. Many models have been proposed, amongst these the most relevant link overvaluation of weight and binge eating with the presence of restrictive eating behavior and with a deficit in managing negative emotions.

According to the transdiagnostic cognitive-behavioral model of eating disorders, as seen above, overvaluation of shape/weight is the driving force that propels engagement in non-compensatory weightcontrol behaviors, such as engagement in restrictive eating and compulsive exercise that is independent of the amount of food intake and presence of binge eating episodes $(1,26)$.

According to this model, low self-esteem, body image-related, generates anxiety and negative emotions resulting in excessive diet restrictions, in turn triggering bingeing.

Popular weight loss strategies among adolescents include dieting and calorie restriction. However, these weight loss techniques put individuals at risk for eating disorders including anorexia nervosa at first, then 
bulimia nervosa (30) and finally also binge eating behavior. It has been proposed that binge eating is largely a product of the particular way that these patients attempt to restrict their eating (i.e., their form of dietary restraint), whether or not there is an actual energy deficit. Rather than adopting general guidelines about how they should eat, they try to adhere to multiple extreme, and highly specific, dietary rules. Accompanying these dietary rules is a tendency to react negatively to the (almost inevitable) breaking of them with even minor dietary slips being interpreted as evidence of their lack of self-control, the result being that they respond by temporarily abandoning their efforts to restrict their eating. Patient reports indicate that this is a major trigger of binge eating with an overcompensation of diet restrictions (31). The peculiar food choice that patients make during binge episodes, in fact, - e.g. fats, carbohydrates, sweets and snacks - has been related to the hypothesis of a "hedonic deprivation", were eating impulsiveness can be triggered by restrictions on palatable foods during everyday life, even if at more recent findings the presence of this clinical feature seems controversial $(1,32)$. Increased engagement in these behaviors leads to binge eating which, in turn, reinforces overvaluation of shape/weight and consequent personal devaluation which rises unpleasant emotions (26).

This therefore maintains the core psychopathology by magnifying patients' concerns about their ability to control their eating, shape and weight. This encourages yet greater dietary restraint, thereby further increasing the risk of binge eating. In the original cognitive behavioral formulation, it was noted that these patients' dietary slips and binges do not happen at random; rather, they are particularly likely to occur in response to acute changes in mood (typically adverse mood states) since these appear to interfere with the ability to maintain dietary restraint. These patients tend to be extremely self-critical, they set themselves demanding standards in terms of their eating, shape and weight, and control. When they cannot meet them, they see themselves as being at fault rather than their standards as being too harsh. The result is a secondary negative self-evaluation. This too maintains the eating disorder since it leads patients to strive even harder to achieve 'success' in the area of life that is most important to them; that is, controlling their eating, shape and weight. In this way a further vicious circle serves to maintain the eating disorder (31). On the other hand, in addition to dietary restraint, deficitary negative emotion managing and emotional eating appear to be involved in development and maintenance of BED. Obese individuals with BED may lack effective strategies for managing negative emotions, and it has been hypothesized that eating is undertaken as a strategy to regulate or change negative emotions (33). Recent studies describe binge eating in BED as the possible result of an immediate breakdown of emotion and impulse regulation caused by sudden increases of negative affect and tension, and/or rapid decrease of positive affect (1).

External emotional eating have been identified as forms of disordered eating behaviors. External eating involves reacting to external cues for food intake (e.g., eating because other people are eating, eating during meal preparation or in response to the sight or smell of food) rather than relying on internal cues, such as hunger. Emotional eating refers to excessive eating in response to emotional experiences such as anxiety or anger. This pattern, linked to attempts to improve one's mood through the consumption of food, has been identified as a precursor to binge eating (30).

It was recognized in the original cognitive behavioral theory that adverse mood states can be a trigger of binge eating, and it was suggested that their primary effect was to disrupt dietary restraint (24). It is now clear that in some patients there is a more complex relationship between emotional states and binge eating. Usually this intolerance is of adverse mood states, such as anger, anxiety or depression, but in some cases there is intolerance of all intense mood states including positive ones (e.g., excitement). Instead of accepting changes in mood and dealing appropriately with them, these patients engage in what may be termed "dysfunctional mood modulatory behavior." This reduces their awareness of the triggering mood state (and the associated cognitions), and also neutralizes it, but at a personal cost. The dysfunctional mood modulatory behaviour may take the form of self-injury (e.g., cutting, punching or burning themselves) which has the effect of rapidly dissipating the initial mood state, or it may involve taking psychoactive substances (e.g., alcohol, tranquillizers) to directly modify how they feel. In patients with bulimia nervosa, binge eating, self-induced vomiting and intense exercising may also be used as forms of mood modulatory behaviour, binge eating most commonly serving this purpose. Among these patients (i.e., those with an eating disorder and mood intolerance), such forms of behaviour (i.e., binge eating, vomiting, intense 
exercising) can become habitual means of mood modulation (31).

It has to be remembered that triggers of binge eating are commonly of interpersonal nature. It can be argued that this reflects a short-term intensification of their need for a sense of "control", a need which is displaced onto dietary self-control. Second, it is obvious that certain interpersonal environments magnify concerns about controlling eating, shape and weight. These include families in which there are other members with an eating disorder, and occupations in which there is pressure to be slim (31). The relationship between interpersonal dynamics and binge behavior is complex and polimorphic, and appears to be strictly related to personality traits and anger. In particular, it has been recently enlighted the role of the personality trait cooperativeness in mediating such relationship. Low cooperativeness, which strictly correlates with the severity of a personality disorder, has been evidenced as a relevant predictor of dropout. Studies demonstrated that coopertiveness not only influence adherence to treatment but is related to binge eating and impulse regulation. In fact many authors underline a strong relationship between anger feelings and interpersonal difficulties, in particular, with regard to interpersonal boundaries and aspects of the experience of the self. Temperamental shyness and sensibility to criticism related to Harm Avoidance may favour anger feelings towards others, which may impair the relational coping skills expressed by Cooperativeness. Social interactions with low Cooperativeness may consequently foster anger feelings and these elicit binge eating and impulsive behaviours when the patient faces with conflicting situations. Therefore, it appears fundamental to address cooperativeness in treating BED: this may rise social skills and social adaptation and help to control anger and, eventually, significantly reduce impulse-related binge symptoms (35).

In conclusion, overvaluation and engagement in non-compensatory weight-control behaviors mutually reinforce each other in a reciprocal manner (26)

\subsubsection{Body dissatisfaction in BED}

Body dissatisfaction is widespread among girls and women throughout Western cultures, with approximately $60 \%$ of $13-15$ year old girls, $90 \%$ of $18-30$ year old women, and $80 \%$ of middle-aged women reporting dissatisfaction with their appearance. Body dissatisfaction appears to be a major risk factor for the development of eating disorders $(36,37)$. Of those individuals who are dissatisfied with their body and wish to alter their appearance, approximately one-third engage in unhealthy body change practices, such as forcing themselves to purge, deliberately abusing laxatives or diuretics, or strict dieting or fasting. Surprisingly, body dissatisfaction is also related to binge eating, and this relationship is not simply driven by shared association with other eating disorder symptoms. The act of binging typically occurs amid a general effort to restrict eating to achieve a slim figure $(30,38)$.

Teasing related to appearance has been one of the most empirically well-supported links to body dissatisfaction and some research supports a causal link between teasing and body dissatisfaction. A metaanalysis suggests that teasing during adolescence may be a risk factor for body dissatisfaction during adulthood (39) and may play a role in the development of body image disturbance and possibly related disordered eating outcomes (40).

It has been shown that subjective perceptions of body weight and, to a lesser extent, body shape, play a greater role in disordered eating and body image concerns than do objective measures of BMI and WHR. In other words, the extent to which someone perceive himself or herself as being overweight is a much better predictor of body dissatisfaction, body esteem, and disordered eating, than the actual BMI (41). Moreover, body dissatisfaction can be evaluated by Digital Photo Distortion Technique, based on an individual photograph of one's own body. The discrepancy scores between the "actual" and "ideal" as well as the "actual" and "felt" body dimensions are higher in obese BED patients compared to non-BED obese patients, indicating a higher degree of discontent with one's ownbody sizeand a greater wish to be thinner than those without an eating disorder.

According to the objectification theory, body dissatisfaction is at least partially linked to the role and position of women in society. Women, particularly in Western cultures, are commonly viewed as objects by society, with their bodies being attended to in a sexual, objectifying manner. Given the pervasiveness of this objectification, it is argued that many women adopt a third-person perspective of their own appearance 
(self-objectification), which, in turn, leads to a preoccupation with outward appearance, more frequent engagement in self-monitoring, and subsequent body dissatisfaction (42).

\subsubsection{How body dissatisfaction may lead to binge eating}

Many theories have been proposed on how body dissatisfaction can contribute to cause and maintain binge eating disorder. Broadbent and colleagues (42) have compared three different models: a dual pathway model involving negative affectivity and dietary restraint, a model considering binge eating as a self-distraction maladaptive coping strategy and a third model based on interoceptive deficits.

The dual pathway model proposes that body dissatisfaction may induce general and intense states of negative affect or depressed mood, which are resolved through distraction and/or comfort through food consumption. Individuals who experience difficulties regulating this emotional arousal may thus use binge eating as a means to distract themselves.

Alternatively, dietary restraint may be used in attempts to reduce body dissatisfaction, but this too leads to overeating as sustained food restriction increases hunger levels, appetitive response to food and, ultimately, increases the likelihood of food over-consumption to restore energy levels after a period of deprivation. The proposed pathways of negative affect and dietary restraint have received extensive support in both eating disordered and non-eating disordered samples of women. Negative mood, depressive symptoms, and emotional regulation difficulties have been shown to be more common among individuals with eating disorders, and in non-clinical populations, these mood disturbances are positively associated with severity of disordered eating symptomatology.

Heatherton and Baumeister's escape from awareness model propose instead that binge eating is a consequence of successful self-distraction attempts (43).

Negative affectivity, a generic feeling of depressed mood or negative mood in relation to body dissatisfaction, may induce some individuals to respond by shifting attention away from considerations of meaning and consequences, and instead move towards emphasis on concrete aspects of stimuli, such as smell, sight, feel, and taste of stimuli in their environment. The focus on these concrete aspects of food (when present) coupled with reduced consideration of the consequences of food consumption may have the effect of temporarily suspending any self-imposed prohibitions on food consumption. Consistent with the escape from awareness model, binge episodes are typically preceded by heightened negative affect, and subsequent to the binge episode, individuals exhibit poor recall of amount of food consumed. Individuals with body dissatisfaction are also more likely to adopt coping strategies involving selfdistraction from, and avoidance of, aversive self-criticism. Moreover, escape from awareness could be favored by a deficit in internal awareness, or interoceptive awareness. According to the Objectification theory, individuals with poor interoceptive awareness are unaware of satiety signals that prevent overeating. Extant literature provides evidence for a link between self-objectification and interoceptive awareness, with lack of awareness being correlated with greater eating disorder symptoms (42).

\subsubsection{Internal weight Bias and self-esteem}

Weight-biased attitudes and resulting weight-stigmatization are prevalent in all domains of society, and individuals with obesity who internalize this bias apply negative stereotypes to themselves and base their self-evaluation on these stigmatizing attitudes. Weight bias internalization among individuals with obesity is associated with a host of negative mental and physical health outcomes such as low self-esteem, weight and shape concerns, body dissatisfaction, and unhealthy eating behaviors including binge eating. Weight bias internalization is distinct from overvaluation of shape and weight because it includes applying negative weight stereotypes to oneself. Low self-esteem, frequently found in BED patients, may contribute to the internalization of weight bias through the process of basing one's self-evaluation strongly in shape and weight $(44,45)$.

The complexity of interactions between body weight and shape, self-esteem, body dissatisfaction, ideal weight and internal weight bias is reflected in the findings that show that such interactions are bi- 
directional. Accordingly, Bucholz and collegues demostrated that internalization of the thin ideal and the muscular ideal, in females and males respectively, has emerged as an important risk factor for the development of body image concerns and eating disorders. Such internalization is culturally dependent and largely influenced by media, especially in adolescents $(30,46)$. Moreover, overweight/obese college women with binge eating showed more distress and preoccupation regarding their shape and weight and lower overall appearance satisfaction and self-esteem in comparison to women without binge eating (6).

\subsubsection{How internal weight bias and low self-esteem may lead to binge eating}

Body esteem and internal weight bias are important factors to consider in understanding the relationship between internalization of the ideal body figure and disordered eating. Body self-esteem can be conceptualized as composed by weight-esteem and appearance-esteem. Studies has shown the latter to be a strong mediator in the relationship between internalization of the ideal body shape and both emotional and external eating. Appearance-esteem also resulted a significant predictor for both emotional eating and external eating in males and females adolescents. Because neuroticism has been linked with emotional and external eating $(47,48)$ it could be that poor appearance-esteem and internalization of the muscular ideal are more likely to occur among males who experience negative affect and emotional instability $(49,50)$. Furthermore, weight-esteem emerged as a mediator in the relationship between internalization of the ideal body shape and emotional eating in adolescents females but not in adolescents males. On the contrary, it appeared to be a major predictor of restrained eating both in adolescents females and males (30). Low self-esteem has not to be considered only as a causative factor, but also a maintenance factor. According to the transdiagnostic cognitive-behavioral model of eating disorders, low self-esteem tends to obstruct change through two main mechanisms. First, it creates in patients hopelessness about their capacity to change, thereby undermining their compliance with treatment; and second, it results in them pursuing, with particular determination, achievement in their valued domains (in this case the pursuit of control over eating, shape and weight) thereby making change in these areas all the more difficult. The state is also self-perpetuating since these patients show particularly pronounced negative cognitive processing biases, coupled with over-generalization, with the result that any perceived "failure" is interpreted as confirmation that they are failures as people thereby reaffirming their overall negative view of themselves (31).

\subsection{The role of body image in the prevention and treatment of eating disorders}

Negative body image is an important risk factor which increases the probability of developing other key components of eating pathology: dietary restraint, binge-eating, and negative affect. Stice (51) conducted a meta-analytic review: data from these studies confirm that negative body image is an independent predictor of disordered eating. Therefore, it is possible to state that, if negative body image could be prevented, the incidence of eating pathology would be reduced (52).

Self compassion is, on the other hand, a psychological dimension that can be protective against the development of binge eating disorder. It is a multi-dimensional construct based on the recognition that suffering, failure, and inadequacy are part of the human condition, and that all people — oneself includedare worthy of compassion. It can be considered as an "adaptive affect regulation" and a coping strategy. Self-compassion may directly mitigate the maladaptive outcomes of poor body image or eating pathology and may prevent the initial occurrence of a risk factor (e.g., thin-ideal internalization) of a maladaptive outcome (e.g., eating pathology), disrupting the vicious circle through which risk factors operate. A recent review conducted by Tosca and collegues strongly support a role for self-compassion as a protective factor in relation to negative body image and ED and may foster other protective factors and potentiate bodyand ED-related outcomes. This finding can be implemented in treatment: self-compassion training may directly or indirectly foster increased interoceptive awareness, and/or be particularly helpful at inducing self-soothing and non-reactivity during unpleasant emotional or endogenous hunger and satiety cues that may otherwise be suppressed or misinterpreted (46). 
Acknowledgement:

Dott. Gabriela Dorati contributed to this chapter

\section{References}

1) Amianto F, Ottone L, Abbate Daga G, Fassino S (2015) Binge-eating disorder diagnosis and treatment: a recap in front of DSM-5. BMC Psychiatry 15: 70 doi: 10.1186/s12888-015-0445-6 PMCID: PMC4397811

2) Amianto F, Lavagnino L, Abbate-Daga G, Fassino S (2011) The forgotten psychosocial dimension of the obesity epidemic. Lancet. 19;378(9805):e8 doi: 10.1016/S0140-6736(11)61778-9

3) Wang YC, McPherson K, Marsh T, Gortmaker SL, Brown M (2011) Health and economic burden of the projected obesity trends in the USA and the UK. Lancet 27;378(9793):815-25 doi: 10.1016/S0140-6736(11)60814-3

4) Hudson JI, Coit CE, Lalonde JK, Pope HG Jr (2010) By how much will the proposed new DSM-5 criteria increase the prevalence of binge eating disorder? Int J Eat Disord. 45(1):139-41 doi: 10.1002/eat.20890

5) Trace SE, Thornton LM, Root TL, Mazzeo SE, Lichtenstein P, Pedersen NL, Bulik CM (2012) Effects of reducing the frequency and duration criteria for binge eating on lifetime prevalence of bulimia nervosa and binge eating disorder: implications for DSM-5. Int J Eat Disord. 45(4):531-6 doi: 10.1002/eat.20955

6) Herbozo S, Schaefer LM, Thompson JK (2015) A comparison of eating disorder psychopathology, appearance satisfaction, and self-esteem in overweight and obese women with and without binge eating. Eat Behav. 17:86-9 doi: 10.1016/j.eatbeh.2015.01.007

7) Bulik CM, Sullivan PF, Kendler KS (2002) Medical and psychiatric morbidity in obese women with and without binge eating. Int J Eat Disord. 32(1):72-8

8) Wilfley DE, Wilson GT, Agras WS (2003) The clinical significance of binge eating disorder. Int J Eat Disord. 34 Suppl:S96-106

9) Heatherton TF, Polivy J, Herman CP, Baumeister RF (1993) Self-awareness, task failure, and disinhibition: how attentional focus affects eating. J Pers. 61(1):49-61

10) Decaluwé V, Braet C, Fairburn CG (2003) Binge eating in obese children and adolescents. Int J Eat Disord. 33(1):78-84

11) Fairburn CG, Beglin SJ (1994) Assessment of eating disorders: interview or self-report questionnaire? Int J Eat Disord.16(4):363-70

12) DSM-IV American Psychiatric Association, 1994 
13) von Ranson KM, Klump KL, Iacono WG, McGue M (2005) The Minnesota Eating Behavior Survey: a brief measure of disordered eating attitudes and behaviors. Eat Behav. 6(4):373-92

14) Van Strien T, Rookus MA, Bergers GP, Frijters JE, Defares PB (1986) Life events, emotional eating and change in body mass index. Int J Obes. 10(1):29-35

15) Amianto F, Northoff G, Abbate Daga G, Fassino S, Tasca GA (2016) Is Anorexia Nervosa a Disorder of the Self? A Psychological Approach. Front Psychol. 7: 849 doi: 10.3389/fpsyg.2016.00849 PMCID: PMC4906654

16) Schaefer M, Northoff G (2017) Who Am I: The Conscious and the Unconscious Self. Front. Hum. Neurosci. https://doi.org/10.3389/fnhum.2017.00126

17) Higgins ET (1987) Self-discrepancy: a theory relating self and affect. Psychol Rev. 94(3):319-40

18) Arciszewski T, Berjot S, Finez L (2012) Threat of the thin-ideal body image and body malleability beliefs: effects on body image self-discrepancies and behavioral intentions. Body Image 9(3):33441 doi: 10.1016/j.bodyim.2012.04.007

19) Valtolina GG (1998) Body-size estimation by obese subjects. Percept Mot Skills. 86(3):1363-74.

20) Glasofer DR, Haaga DA, Hannallah L, Field SE, Kozlosky M, Reynolds J, Yanovski JA, Tanofsky-Kraff M (2013) Self-efficacy beliefs and eating behavior in adolescent girls at-risk for excess weight gain and binge eating disorder. Int J Eat Disord. 46(7):663-8 doi: 10.1002/eat.22160

21) Emily M. Pisetsky, Ann F. Haynos, Jason M. Lavender, Scott J. Crow, Carol B. Peterson (2017) Associations between emotion regulation difficulties, eating disorder symptoms, non-suicidal selfinjury, and suicide attempts in a heterogeneous eating disorder sample. Comprehensive Psychiatry $73: 143-150$

22) Mason TB, Lavender JM, Wonderlich SA, Crosby RD, Engel SG, Strauman TJ, Mitchell JE, Crow SJ, Le Grange D, Klein MH, Smith TL, Peterson CB (2016) Self-Discrepancy and Eating Disorder Symptoms Across Eating Disorder Diagnostic Groups. Eur Eat Disord Rev. 24(6):541545 doi: $10.1002 /$ erv. 2483

23) Monell E, Högdahl L, Mantilla EF, Birgegård A (2015) Emotion dysregulation, self-image and eating disorder symptoms in University Women. J Eat Disord. 1;3:44 doi: 10.1186/s40337-0150083-x

24) Cooper Z, Fairburn CG (2003) Refining the definition of binge eating disorder and nonpurging bulimia nervosa. Int J Eat Disord. 34 Suppl:S89-95

25) Fairburn CG, Cooper Z, Doll HA, O'Connor ME, Bohn K, Hawker DM, Wales JA, Palmer RL (2009)Transdiagnostic cognitive-behavioral therapy for patients with eating disorders: a two-site trial with 60-week follow-up. Am J Psychiatry 166(3):311-9 doi: 10.1176/appi.ajp.2008.08040608

26) Tabri N, Murray HB, Thomas JJ, Franko DL, Herzog DB, Eddy KT (2015) Overvaluation of body shape/weight and engagement in non-compensatory weight-control behaviors in eating 
disorders: is there a reciprocal relationship? Psychol Med. 45(14):2951-8 doi:

$10.1017 / \mathrm{S} 0033291715000896$

27) Grilo CM, Crosby RD, Masheb RM, White MA, Peterson CB, Wonderlich SA, Engel SG, Crow SJ, Mitchell JE (2009) Overvaluation of shape and weight in binge eating disorder, bulimia nervosa, and sub-threshold bulimia nervosa. Behav Res Ther. 47(8):692-6 doi:

10.1016/j.brat.2009.05.001

28) Grilo CM, White MA, Gueorguieva R, Wilson GT, Masheb RM (2013) Predictive significance of the overvaluation of shape/weight in obese patients with binge eating disorder: findings from a randomized controlled trial with 12-month follow-up. Psychol Med. 43(6):1335-44 doi: $10.1017 / \mathrm{S} 0033291712002097$

29) Ojserkis R, Sysko R, Goldfein JA, Devlin MJ (2012) Does the overvaluation of shape and weight predict initial symptom severity or treatment outcome among patients with binge eating disorder? Int J Eat Disord. 45(4):603-8 doi: 10.1002/eat.20981

30) Flament MF, Hill EM, Buchholz A, Henderson K, Tasca GA, Goldfield G (2012) Internalization of the thin and muscular body ideal and disordered eating in adolescence: the mediation effects of body esteem. Body Image 9(1):68-75 doi: 10.1016/j.bodyim.2011.07.007

31) Fairburn CG, Cooper Z, Shafran R (2003) Cognitive behaviour therapy for eating disorders: a "transdiagnostic" theory and treatment. Behav Res Ther. 41(5):509-28

32) Wolfe BE, Baker CW, Smith AT, Kelly-Weeder S (2009) Validity and utility of the current definition of binge eating. Int J Eat Disord. 42(8):674-86 doi:10.1002/eat.20728

33) Gianini LM, White MA, Masheb RM (2013) Eating pathology, emotion regulation, and emotional overeating in obese adults with Binge Eating Disorder. Eat Behav. 14(3):309-13 doi: 10.1016/j.eatbeh.2013.05.008

34) Cooper PJ, Fairburn CG (1986) The depressive symptoms of bulimia nervosa. Br J Psychiatry148:268-74

35) Amianto F, Siccardi S, Abbate-Daga G, Marech L, Barosio M, Fassino S (2012) Does anger mediate between personality and eating symptoms in bulimia nervosa? Psychiatry Res. 30;200(23):502-12 doi: 10.1016/j.psychres.2012.07.036

36) Polivy J, Herman CP (2002) Causes of eating disorders. Annu Rev Psychol. 53:187-213

37) Stice E, Schupak-Neuberg E, Shaw HE, Stein RI (1994) Relation of media exposure to eating disorder symptomatology: an examination of mediating mechanisms. J Abnorm Psychol.103(4):836-40

38) Holmes M, Fuller-Tyszkiewicz M, Skouteris H, Broadbent J (2015) Understanding the link between body image and binge eating: a model comparison approach. Eat Weight Disord. 
20(1):81-9 doi: 10.1007/s40519-014-0141-4

39) Grilo CM, Wilfley DE, Brownell KD, Rodin J (1994) Teasing, body image, and self-esteem in a clinical sample of obese women. Addict Behav. 19(4):443-50

40) Menzel JE, Schaefer LM, Burke NL, Mayhew LL, Brannick MT, Thompson JK (2010) Appearance-related teasing, body dissatisfaction, and disordered eating: A meta-analysis. Body Image 7(4):261-70 doi: 10.1016/j.bodyim.2010.05.004

41) Wilson JM, Tripp DA, Boland FJ (2005) The relative contributions of subjective and objective measures of body shape and size to body image and disordered eating in women. Body Image. 2(3):233-47

42) Holmes M, Fuller-Tyszkiewicz M, Skouteris H, Broadbent J (2015) Understanding the link between body image and binge eating: a model comparison approach. Eat Weight Disord. 20(1):81-9 doi: 10.1007/s40519-014-0141-4

43) Heatherton TF, Baumeister RF (1991) Binge eating as escape from self-awareness. Psychol Bull. 110(1):86-108

44) Pearl RL, White MA, Grilo CM (2014) Overvaluation of shape and weight as a mediator between self-esteem and weight bias internalization among patients with binge eating disorder. Eat Behav. 15(2):259-61 doi: 10.1016/j.eatbeh.2014.03.005

45) Lewer M, Nasrawi N, Schroeder D, Vocks S (2016) Body image disturbance in binge eating disorder: a comparison of obese patients with and without binge eating disorder regarding the cognitive, behavioral and perceptual component of body image Eat Weight Disord. 21(1):115-25 doi: 10.1007/s40519-015-0200-5

46) Braun TD, Park CL, Gorin A (2016) Self-compassion, body image, and disordered eating: A review of the literature. Body Image 17:117-31 doi: 10.1016/j.bodyim.2016.03.003

47) Heaven PC, Mulligan K, Merrilees R, Woods T, Fairooz Y (2001) Neuroticism and conscientiousness as predictors of emotional, external, and restrained eating behaviors. Int J Eat Disord. 30(2):161-6

48) Steptoe A, Pollard TM, Wardle J (1995) Development of a measure of the motives underlying the selection of food: the food choice questionnaire. Appetite 25(3):267-84

49) Amianto F, Dorati G, Spalatro A, Abbate-Daga G, Fassino S (2016) Homosexuality and Anorexia Nervosa: An Explorative Study on Personality Traits. Acta Psychopathol. 2:42. doi: $10.4172 / 2469-6676.100068$

50) Fassino S, Abbate-Daga G, Leombruni P, Amianto F, Rovera G, Rovera GG (2001) Temperament and character in italian men with anorexia nervosa: a controlled study with the temperament and 
character inventory. J Nerv Ment Dis. 189(11):788-94

51) Stice E (2002) Risk and maintenance factors for eating pathology: a meta-analytic review. Psychol Bull. 128(5):825-48

52) Levine MP, Piran N (2004) The role of body image in the prevention of eating disorders. Body Image 1(1):57-70 